\title{
La gestión del conocimiento en instituciones educativas
}

\author{
Knowledge management in educational institutions \\ Gestão do conhecimento em instituições de ensino
}

Jesús Antonio Álvarez-Cedillo

Instituto Politécnico Nacional - UPIICSA, México jaalvarez@ipn.mx

https://orcid.org/0000-0003-0823-4621

Mario Aguilar-Fernández

Instituto Politécnico Nacional - UPIICSA, México

maguilarfer@ipn.mx

https://orcid.org/0000-0003-2621-8692

Teodoro Álvarez-Sánchez

Instituto Politécnico Nacional - CITEDI, México

talvarezs@ipn.mx

https://orcid.org/0000-0002-2975-7125

Brenda García Jarquín

Instituto Politécnico Nacional CDMX - ZACATENCO, México jarquin_garcia@yahoo.com.mx https://orcid.org/0000-0001-7192-2342

Julián Patiño Ortiz

Instituto Politécnico Nacional CDMX - ZACATENCO, México jpatinoo@ipn.mx https://orcid.org/0000-0001-8106-9293 


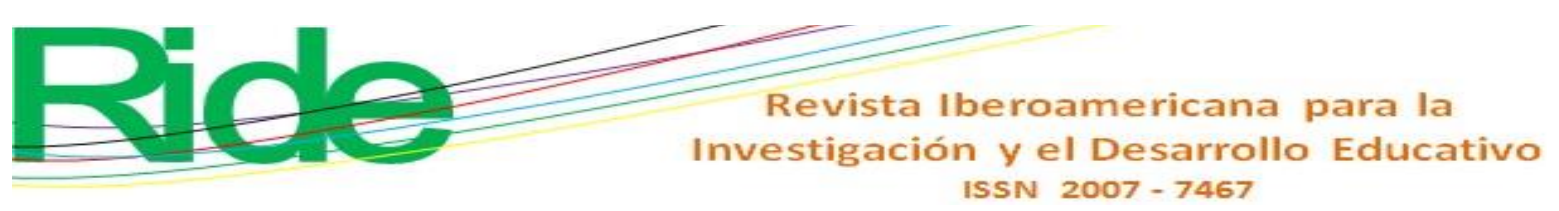

measurement index, in different Mexican educational institutions. The result achieved is 67.39\%, which represents great challenges in terms of scientific production, mainly.

Keywords: knowledge, explicit knowledge, knowledge management, memory index of educational organizational intelligence, educational institutions.

\section{Resumo}

A tarefa fundamental da educação é permitir que o ser humano seja capaz de se conduzir na vida e interagir com o próximo da forma mais amigável possível. Por isso, a instituição educacional tem que modificar algumas das práticas que, se eram necessárias na época de Humboldt, hoje são francamente inoperantes para o mesmo desenvolvimento. As instituições educacionais e a sociedade não podem viver separadas, por isso têm que se abrir, se a sociedade do conhecimento afirma sê-lo. Esse contato permanente entre saberes e práticas sociais é que deve ser o objetivo fundamental da educação permanente. Neste artigo, portanto, mostra-se a importância de agregar a gestão do conhecimento às instituições de ensino de pós-graduação e à pesquisa, por meio de uma exploração quantitativa onde se destacam seu uso, sua perspectiva e suas consequências observáveis em uma amostra de 3.000. pessoas. O objetivo foi avaliar a gestão do conhecimento por meio do cálculo do índice de medição da inteligência organizacional em diferentes instituições de ensino mexicanas. O resultado alcançado foi de $67,39 \%$, o que representa grandes desafios em termos de produção científica, principalmente.

Palavras-chave: conhecimento, conhecimento explícito, gestão do conhecimento, índice de memória de inteligência organizacional educacional, instituições educacionais.

Fecha Recepción: Septiembre 2019

Fecha Aceptación: Septiembre 2020 


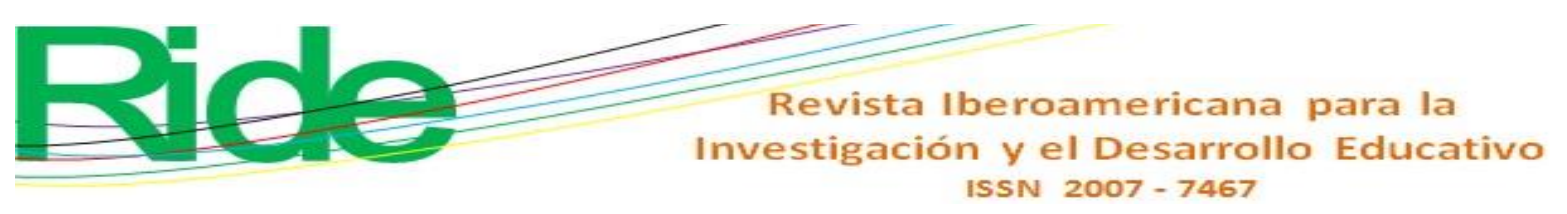

\section{Introducción}

La gestión del conocimiento (GC) — conocida como knowledge management (Rubinstein, 2005) - busca transferir el conocimiento explícito y usarlo como un recurso disponible para otros en la misma organización. Este proceso implica desarrollar técnicas para capturar, organizar y almacenar el conocimiento, utilizando a los colaboradores para transformarlo como un activo intelectual que preste beneficios y pueda compartirse.

En la actualidad, las tecnologías de la información y comunicación (TIC) permiten contar con herramientas que promueven la gestión del conocimiento en las empresas, lo cual se sustenta básicamente en la recolección, la transferencia, la seguridad y la administración sistemática de información, junto con un sistema diseñado para ayudar a hacer el mejor empleo de ese conocimiento (Correa, Gómez y Cano, 2010).

Su uso va más allá de la tecnología de la información, pues todos los integrantes de una organización — desde el más alto directivo hasta el puesto más bajo, en cualquier área — aportan conocimientos y experiencias (Aguilera y Riascos, 2009). La gestión del conocimiento está formada por los siguientes elementos esenciales (Hernández, Marulanda y López, 2014):

- Edificar, recoger y organizar el conocimiento existente para facilitar la creación de nuevo conocimiento.

- Apuntar a la innovación.

- Reutilizar el conocimiento.

A través del uso de la gestión del conocimiento se desarrolla la habilidad de la gente que se apoya en la organización para lograr un mejor desempeño. La gestión del conocimiento crece informalmente por medio de discusiones y sesiones que representan, de manera formal, los programas de capacitación como práctica emergente. A partir de la alta gestión del conocimiento, la institución representa el vocero principal y oficial del conocimiento, y de él depende el establecimiento de planes corporativos y de otras prácticas (Pávez, 2000).

El término institución, por otra parte, se utiliza para poder aludir a ciertas normas que expresan valores ante una realidad social (Valdivieso, 2001). Se utilizan cuatro conceptos para poder definir una institución:

- La institución es un sinónimo de regularidad social que alude a la aplicación de normas y presenta valores sociales. El individuo siempre tiene carácter dentro del grupo y es controlado por diferentes regulaciones internas y externas. 


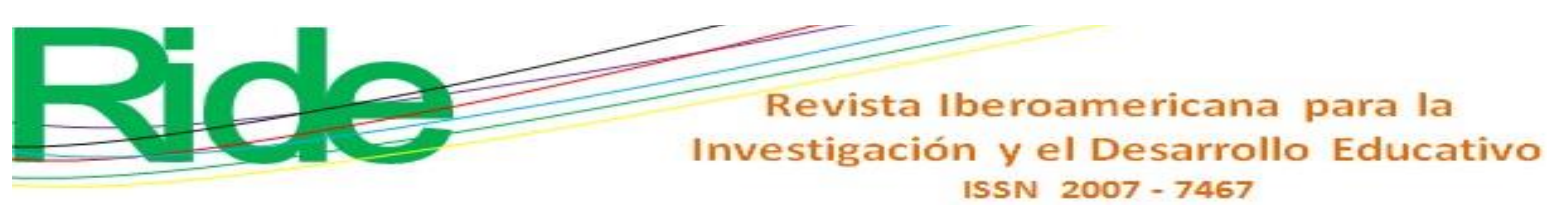

- La institución como sinónimo de establecimiento a la organización. Expresa una función especializada que cuenta con su espacio propio.

- La institución como un ente ante la existencia del mundo simbólico.

- La institución social: ente que marca lo permitido, lo prohibido, el reconocimiento, la obediencia.

Hoy se reconoce que las instituciones son los mecanismos fundamentales para el desarrollo de nuestra sociedad, las cuales se encuentran regidas por reglas generales que permiten la coordinación de los sujetos y la gestión de los conflictos (Hodgson, 2011).

Las instituciones educativas se enfocan en la parte social sobre la formación integral del individuo, el fortalecimiento de los valores tradicionales y el mejoramiento del individuo para ser una persona mejor (Representación de la Unesco en Perú, 2011). Se caracterizan, en forma inadecuada, por diagnosticar y gestionar sobre el entorno de una cultura organizacional donde escasamente se valora al personal, al aprendizaje, al trabajo en equipo y al conocimiento.

Carece de una visión compartida para lograr la misión, la visión y el compromiso institucional de todas las instancias (desde la dirección), y generalmente falla en la coordinación y en el desarrollo de estrategias que estimulen y motiven al personal para desarrollar su capacidad productiva.

Explicado lo anterior, se puede decir que el objetivo de la presente investigación es evaluar la gestión del conocimiento a través del cálculo del índice de medición de inteligencia organizacional en distintas instituciones educativas mexicanas.

\section{Gestión del conocimiento como estrategia competitiva en las instituciones educativas}

El conocimiento como recurso se ha convertido en la estrategia competitiva para las naciones, las instituciones educativas y las personas. En la sociedad actual se ha producido un cambio tangible en la naturaleza e importancia del conocimiento y sus relaciones con la prosperidad (Everett, Geoffrey y Carl, 2006).

Los contrastes básicos que otorgan ventajas económicas a países, asociaciones e individuos durante períodos más amplios terminan siendo aquellos que dependen de aptitudes e información, y se les denomina recursos evasivos o capital académico (Kabir, 2013; Rubinstein, 2005). 


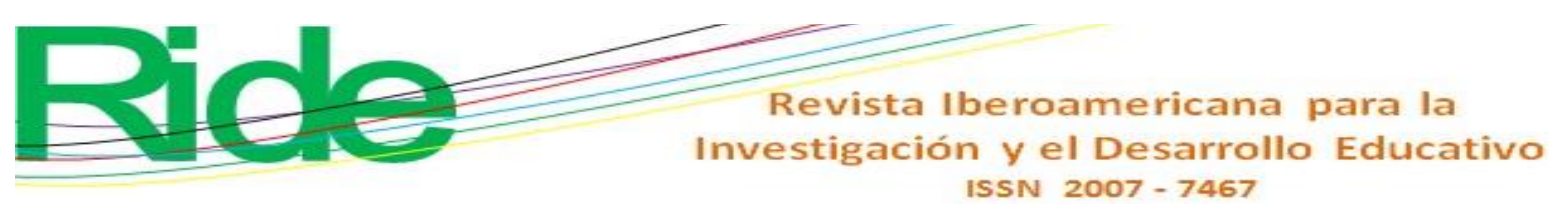

Las tecnologías de la información (TI) han permitido cambiar la forma de vida, las conexiones y la manera en que trabajan las personas. La sociedad de datos y la sociedad del aprendizaje se representan pensando en la información como el punto focal de los diversos ejercicios de individuos y asociaciones para generar rentabilidad (Coll, 2009). La sociedad del aprendizaje, la edad, la preparación y el cambio de datos en información útil se convierten en las fuentes esenciales de rentabilidad y poder.

Debido a los avances extraordinarios en datos e innovaciones de intercambios, las nuevas conexiones entre individuos y organizaciones se agrupan como "sistemas" que surgen sin inhibiciones y se reconfiguran continuamente (Avendaño-Villa, Cortés-Peña y Guerrero-Cuentas, 2015). La Network Society permite construir varias redes individuales que dependen de intereses individuales o afinidades individuales (Castells, 1997). El sistema hace posible que las personas con actividades comparativas fortalezcan sus sentimientos y establezcan comunidades informales innovadoras.

En la sociedad del aprendizaje actual, la información es explícitamente el factor y el margen de maniobra que permite la edad y la capacidad de apoyo de las asociaciones en la economía actual para reaccionar ante las dificultades que la nueva sociedad demanda hoy (Racionero y Serradell, 2005). En los fundamentos actuales de enseñanza, debido a las progresiones que en la última década se han producido, el avance de la nueva correspondencia y los progresos en los datos han dado a las organizaciones una capacidad y productividad más prominentes para tratar con su capital académico solo para crear nuevos beneficios, artículos, patentes, emprendimientos y el desarrollo de otra metodología de administración (Begoña, 2008).

Las instituciones educativas están cambiando sin duda la forma en que la economía de las organizaciones funciona en este mundo globalizado. Inteligentemente, no todas las naciones han distinguido oportunidades similares para presentar esta forma de pensar, en vista de la administración de recursos evasivos para mezclar el aprendizaje y aumentar la rivalidad y la estimación del mercado de las organizaciones en México (Minakata, 2009). 


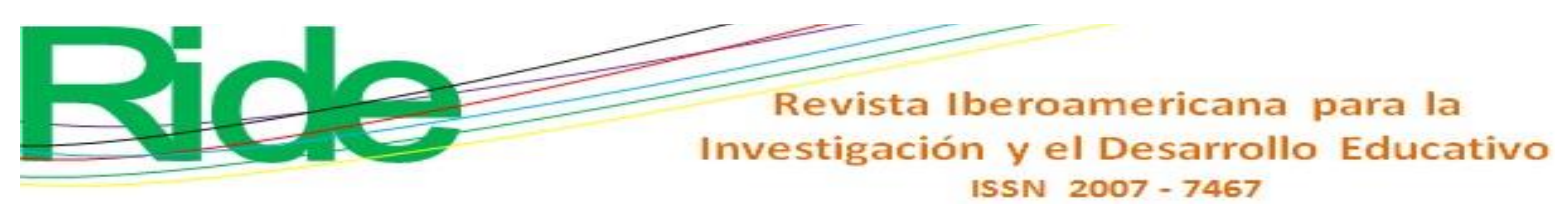

\section{Revisión de investigaciones previas}

De los múltiples documentos que han sido escritos sobre la gestión del conocimiento en instituciones de educación superior, se podrían mencionar el trabajo de Alfonzo (2018) —quien explica la importancia de la gestión del conocimiento para el fortalecimiento de instituciones educativas-, así como las investigaciones de Lara $(2015,2016)$, quien se enfoca en la gestión del conocimiento como facilitador de tecnología y como una herramienta didáctica y educativa para enseñar y aprender colaborativamente. Barroso (2011), por su parte, presenta un trabajo con doble intención, pues a) determina la manera de adquirir, usar y transferir el conocimiento por parte de instituciones de educación superior y centros de investigación científica en el estado de Yucatán, y b) ofrece una evaluación de la gestión del conocimiento de dichas instituciones, a través de una escala de competencias.

Alzate (2015), por otra parte, analiza la gestión del conocimiento como un desafío para las instituciones educativas en Colombia, incluyendo la convergencia interdisciplinar de otros campos de conocimiento. Naranjo, González y Rodríguez (2016) utilizan la gestión del conocimiento como marco para el diseño de estrategias para que las IES se instauren como organizaciones del conocimiento. Moscoso-Zea y Luján-Mora (2017) describen una guía práctica para la implementación de la gestión del conocimiento en instituciones de educación superior. CorreaDíaz, Benjumea-Arias y Valencia-Arias (2019) estudian la manera en que la gestión del conocimiento facilita la mejora de situaciones problemáticas educativas en la actual economía.

Otra serie de documentos — que como finalidad han medido la gestión del conocimiento en distintas organizaciones - son los siguientes: Lobato-Baez, Morales-Rosales, Toriz-Palacios y López-Arciga (2018) proponen la construcción de un modelo de gestión del conocimiento para la industria del software en México, en el que recopilan indicadores a partir de la aplicación de la matriz de índice de medición de inteligencia organizacional (IMIO). Bernal, Torres, Turriago y Sierra (2010) miden la gestión del conocimiento en distintas organizaciones con la matriz IMIO.

\section{Diseño metodológico}

El presente estudio es de índole cuantitativa y de carácter exploratorio, y fue realizado con una muestra de 3000 personas (60\% hombres y $40 \%$ mujeres. De todos ellos, $20 \%$ son directivos y mandos medios, y $80 \%$ profesores, entre los 30 y 75 años de edad, con una antigüedad laboral de 20 años en promedio) de doce instituciones educativas de diferentes tamaños (grandes, medianas y pequeñas). La aplicación del instrumento seleccionado fue una encuesta durante el periodo 


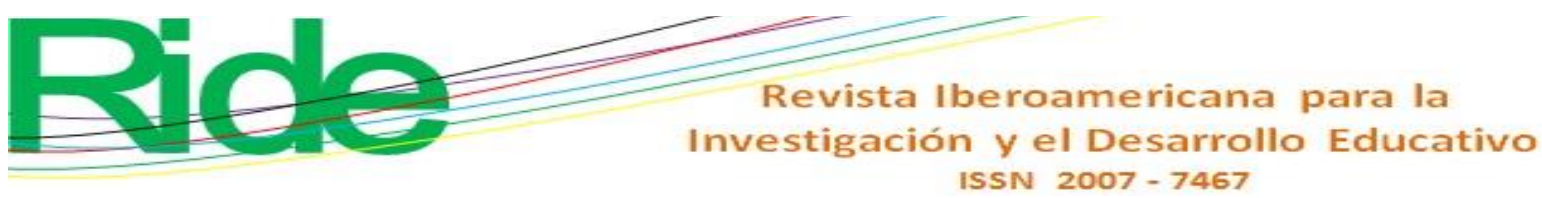

comprendido entre los meses de febrero a octubre de 2019. La encuesta quedó conformada por dos partes:

- Nivel de importancia del aprendizaje: Se compone de un estudio que comprende doce explicaciones identificadas con el nombramiento, la aplicación y la edad de aprendizaje en la instrucción en relación con la preparación, el examen ecológico y la utilización de avances y correspondencias de datos. Esta revisión se planificó explícitamente para esta investigación y está organizada por una escala Likert (Spooren, Mortelmans y Denekens, 2007), donde el encuestado calificó cada anuncio como lo indica su discernimiento, en puntajes del 1 al $4(1=$ fuertemente desviado, $2=$ parcialmente diferente, 3 = parcialmente concurrente y 4 = totalmente concurrente).

- Red del índice de medición de inteligencia organizacional, constituida por tres medidas o variables: la memoria autorizada, la red básica de límites y cultura, marcos mentales y prácticas. Del mismo modo, cada medición está compuesta por varias articulaciones que se muestran en los cuadros internos de la cuadrícula particular, y se puntúan mediante una escala de 1 a $4(1=$ malo, $2=$ regular, $3=$ bueno y $4=$ excelente $)$.

\section{Metodología para cálculo del índice de medición de inteligencia organizacional}

La matriz del índice de medición de inteligencia organizacional es un instrumento estructurado por Arboníes y Aldazabal (2005), que tiene el objetivo de evaluar la gestión del conocimiento en las organizaciones (Martínez y Martínez, 2010). Para calcular esta lista, se definieron las siguientes variables:

1. Se realiza el cálculo de la generación de valor a partir de las capacidades de la organización (GVACO). Se consideraron las diferentes capacidades que condicionan en diferente proporción la generación de valor:
a) Capacidad de vigilar (CV);
b) Capacidad de responder (CR);
c) Capacidad de resolver (CRP);
d) Capacidad de aprender (CA);
e) Capacidad de innovar (CI);
f) Capacidad para explotación del conocimiento (CEC). 


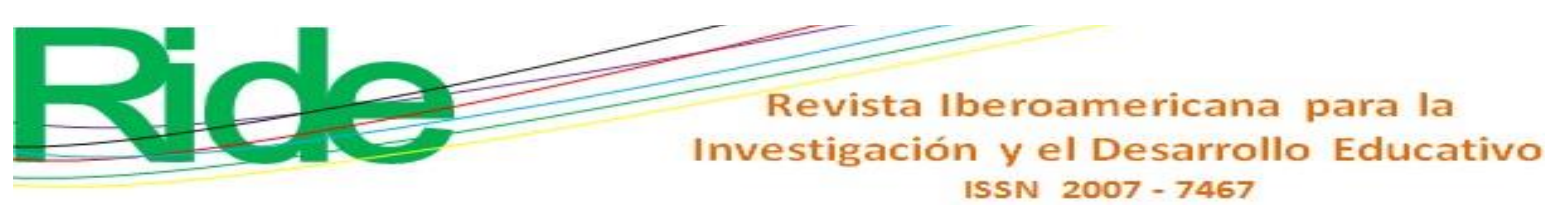

2. Generación de valor tomando como base los K-facts: Se realiza la identificación y la evaluación de los flujos de conocimiento en las instituciones educativas (GVAPE). Esto se determina para los puntos de vista internos y externos, que están interrelacionados para la satisfacción de los destinos vitales de la asociación. Se consideraron las capacidades que condicionan en diferente proporción la generación de valor:

a) Generación de valor a partir de las perspectivas de la organización capacidad de vigilar (GVAPE);

b) Proyectos (Po);

c) Patentes $(\mathrm{Pa})$;

d) Alumnos graduados (Ag);

e) Vinculaciones (Vi);

f) Capítulos de libro (CL);

g) Congresos y simposios (Co).

3. Generación de valor a partir de la memoria organizativa/capital estructural GVAMO. Los elementos identificados con memoria autorizada demuestran la edad dados los puntos de vista internos y externos, según se persigue: GVAMO depende de Po (proyectos), $\mathrm{Pu}$ (publicaciones), $\mathrm{Pa}$ (patentes), Ag (estudiantes graduados), Vi (enlaces), CL (secciones de libros), Co (congresos y simposios).

4. Cálculo de la generación de valor a partir de la cultura, aptitudes y comportamiento organizacional GVACUL. Los aspectos relacionados con la cultura, aptitudes y comportamiento organizacional evidencian la generación de acuerdo con las perspectivas internas y externas. Está en función de Po (proyectos), Pu (publicaciones), Pa (patentes), Ag (alumnos graduados), Vi (vinculaciones), CL (capítulos de libro), Co (congresos y simposios).

A partir de estos cuatro componentes de generación de valor, se tiene que el índice de memoria organizacional (IMIO) está en función de:

- GVACO: Generación de valor a partir de las capacidades de la organización.

- GVAPE: Generación de valor a partir de las perspectivas de la organización.

- GVAMO: Generación de valor a partir de la memoria organizativa.

- GVACUL: Generación de valor a partir de la cultura, aptitudes y comportamiento organizacional.

De esta forma, el IMIO se determinará en función de estos cuatro valores de acuerdo a la ecuación 1. 


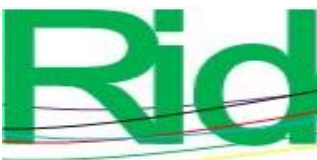

Revista Iberoamericana para la Investigación y el Desarrollo Educativo

$$
I M I O=f(G V A C O+G V A P E+G V A M O+G V A C U L)(1)
$$

El cálculo de la matriz consiste en completar cada celda, con la escala mencionada anteriormente: mala, regular, buena o excelente.

\section{Análisis e interpretación de los resultados}

Al calificar completamente la matriz, se llega a uno de los generadores de valor, representado por un color que refleja el estado en que se encuentra la respuesta. Lo anterior se clasifica de este modo:

- Verde: No hay distorsiones o rupturas entre capacidades y perspectivas, ya que se encuentran en un estado con una capacidad entre $76 \%$ y $100 \%$.

- Amarillo: Las organizaciones educativas están en dificultades relevantes; se está en una capacidad entre $51 \%$ y $75 \%$, donde se corre el riesgo de no tener capacidades frente a las perspectivas o viceversa, y es una alerta sobre las posibilidades de mejoras bien sea de perspectivas o de capacidades.

- Rojo: Las instituciones educativas presentan distorsiones o rupturas entre perspectivas y capacidades críticas, y necesitan atención inmediata, es decir, están muy por debajo de $50 \%$ de las capacidades frente a las perspectivas.

Esta convención de colores permite analizar los aspectos en los cuales las capacidades no están ofreciendo una respuesta acertada para cada una de las perspectivas analizadas. Además, pueden ser representadas en un dashboard de manera gráfica.

En cuanto a la memoria organizativa y la cultura, se muestran aparte y se analizan relacionando el conocimiento que acumula la organización (memoria) y la respuesta frente a la sociedad (cultura y aptitudes). La tabla 1 muestra los valores obtenidos para la memoria organizativa GVAMO. 

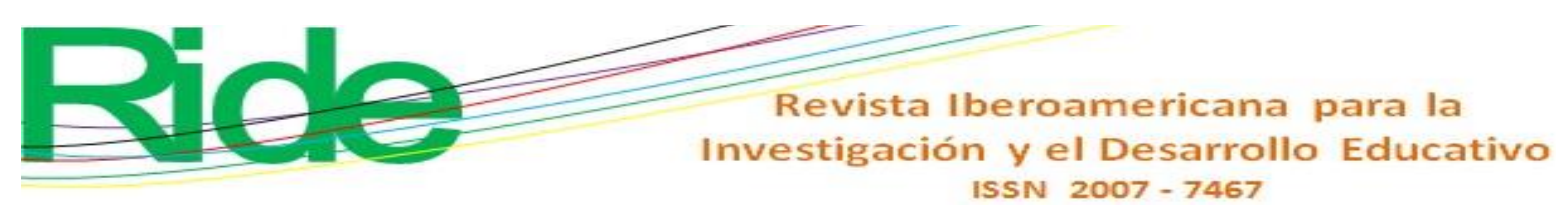

Tabla 1. Capacidades relacionadas con memoria organizativa

\begin{tabular}{|c|c|c|c|c|c|c|c|}
\hline $\begin{array}{c}\text { Proyect } \\
\text { os }\end{array}$ & $\begin{array}{c}\text { Publicacion } \\
\text { es }\end{array}$ & $\begin{array}{c}\text { Patent } \\
\text { es }\end{array}$ & $\begin{array}{c}\text { Alumnos } \\
\text { graduad } \\
\text { os }\end{array}$ & $\begin{array}{c}\text { Vinculacion } \\
\text { es }\end{array}$ & $\begin{array}{c}\text { Capítul } \\
\text { os de } \\
\text { libro }\end{array}$ & $\begin{array}{c}\text { congres } \\
\text { os }\end{array}$ & $\begin{array}{c}\text { GVAM } \\
\text { O }\end{array}$ \\
\hline $\mathbf{7 0 . 5} \%$ & $69.78 \%$ & 69.53 & $70.5 \%$ & $70.14 \%$ & $70.14 \%$ & $71.12 \%$ & $71.12 \%$ \\
& & $\%$ & & & & \\
\hline
\end{tabular}

Fuente: Elaboración propia

La tabla 2 enseña la relación de las capacidades con proyectos realizados.

Tabla 2. Capacidades relacionadas con proyectos realizados

\begin{tabular}{|c|c|c|c|c|c|c|c|}
\hline & $\mathrm{CV}$ & CR & CRP & CA & CI & CEC & $\begin{array}{l}\text { TOT } \\
\text { AL }\end{array}$ \\
\hline & $\begin{array}{l}\text { CÓMO SE } \\
\text { DESARRO } \\
\text { LLAN }\end{array}$ & $\begin{array}{l}\text { QUÉ } \\
\text { TECNOL } \\
\text { OGÍAS } \\
\text { SON } \\
\text { USADAS }\end{array}$ & $\begin{array}{l}\text { RESOLU } \\
\text { CION DE } \\
\text { PROYEC } \\
\text { TOS }\end{array}$ & $\begin{array}{l}\text { CAPACI } \\
\text { DAD DE } \\
\text { APREN } \\
\text { DER }\end{array}$ & $\begin{array}{l}\text { NUEVOS } \\
\text { CONOCIMI } \\
\text { ENTOS }\end{array}$ & $\begin{array}{l}\text { NUEVO } \\
\text { S } \\
\text { PROYE } \\
\text { CTOS }\end{array}$ & \\
\hline $\begin{array}{l}\text { Proye } \\
\text { ctos }\end{array}$ & $72.12 \%$ & $70.5 \%$ & $71.5 \%$ & $68.5 \%$ & $70.5 \%$ & $60.0 \%$ & $\begin{array}{l}80.6 \\
\%\end{array}$ \\
\hline
\end{tabular}

Fuente: Elaboración propia

La tabla 3 muestra la relación de las capacidades con artículos realizados. 
Tabla 3. Capacidades relacionadas con artículos realizados

\begin{tabular}{|c|c|c|c|c|c|c|c|}
\hline & $\begin{array}{l}\text { CV- } \\
\text { Cap. } \\
\text { Vigilar }\end{array}$ & $\begin{array}{l}\text { CR-Cap. } \\
\text { respuest } \\
\text { a }\end{array}$ & $\begin{array}{l}\text { CRP Cap. } \\
\text { Resolver } \\
\text { problema } \\
\text { S }\end{array}$ & $\begin{array}{l}\text { CA -Cap. } \\
\text { aprender }\end{array}$ & $\begin{array}{l}\text { CI- } \\
\text { Cap. } \\
\text { innovar }\end{array}$ & $\begin{array}{l}\text { CEC-Cap. } \\
\text { Explotación } \\
\text { del } \\
\text { conocimient } \\
\text { o }\end{array}$ & $\begin{array}{l}\text { TOTA } \\
\text { L }\end{array}$ \\
\hline & $\begin{array}{l}\text { Lo que } \\
\text { publica } \\
\text { n otros } \\
\text { centros }\end{array}$ & $\begin{array}{l}\text { Nuevos } \\
\text { campos }\end{array}$ & $\begin{array}{l}\text { Otros } \\
\text { artículos }\end{array}$ & $\begin{array}{l}\text { Artículos } \\
\text { publicado } \\
\text { S }\end{array}$ & $\begin{array}{l}\text { Nuevos } \\
\text { artículo } \\
\text { s }\end{array}$ & Divulgación & \\
\hline $\begin{array}{l}\text { Artículo } \\
\text { S }\end{array}$ & $68.75 \%$ & $68.75 \%$ & $67.19 \%$ & $70.5 \%$ & $69.47 \%$ & $65.27 \%$ & $68.32 \%$ \\
\hline
\end{tabular}

Fuente: Elaboración propia

La tabla 4 muestra la relación de las capacidades con patentes realizadas.

Tabla 4. Capacidades relacionadas con patentes realizadas

\begin{tabular}{|c|c|c|c|c|c|c|c|}
\hline & $\begin{array}{l}\text { CV- } \\
\text { Cap. } \\
\text { vigilar }\end{array}$ & $\begin{array}{l}\text { CR-Cap. } \\
\text { respuesta }\end{array}$ & $\begin{array}{l}\text { CRP Cap. } \\
\text { Resolver } \\
\text { problemas }\end{array}$ & $\begin{array}{l}\text { CA -Cap. } \\
\text { Aprender }\end{array}$ & $\begin{array}{l}\text { CI-Cap. } \\
\text { innovar }\end{array}$ & $\begin{array}{l}\text { CEC-Cap. } \\
\text { Explotación } \\
\text { del } \\
\text { conocimiento }\end{array}$ & Total \\
\hline & $\begin{array}{l}\text { Lo que } \\
\text { publican } \\
\text { otros } \\
\text { centros }\end{array}$ & Nuevos c & $\begin{array}{l}\text { Otras } \\
\text { patentes }\end{array}$ & $\begin{array}{l}\text { Patentes } \\
\text { publicadas }\end{array}$ & $\begin{array}{l}\text { Nuevas } \\
\text { patentes }\end{array}$ & Divulgación & \\
\hline Patentes & $8.75 \%$ & $2.25 \%$ & $67.19 \%$ & $1.5 \%$ & $0.95 \%$ & $65.27 \%$ & $\begin{array}{l}24.31 \\
\%\end{array}$ \\
\hline
\end{tabular}

Fuente: Elaboración propia

La tabla 5 muestra la relación de las capacidades con alumnos graduados. 
Tabla 7. Capacidades relacionadas con capítulos de libro realizados

\begin{tabular}{|c|c|c|c|c|c|c|c|}
\hline & $\begin{array}{l}\text { CV- } \\
\text { Cap. } \\
\text { vigilar }\end{array}$ & $\begin{array}{l}\text { CR-Cap. } \\
\text { respuest } \\
\text { a }\end{array}$ & $\begin{array}{l}\text { CRP Cap. } \\
\text { Resolver } \\
\text { problema } \\
\text { s }\end{array}$ & $\begin{array}{l}\text { CA -Cap. } \\
\text { aprender }\end{array}$ & $\begin{array}{l}\text { CI-Cap. } \\
\text { innovar }\end{array}$ & $\begin{array}{l}\text { CEC-Cap. } \\
\text { Explotación } \\
\text { del } \\
\text { conocimient } \\
\text { o }\end{array}$ & TOTAL \\
\hline & $\begin{array}{l}\text { Lo que } \\
\text { publican } \\
\text { otros } \\
\text { centros }\end{array}$ & $\begin{array}{l}\text { Nuevos } \\
\text { campos }\end{array}$ & $\begin{array}{l}\text { Otros } \\
\text { capitulos }\end{array}$ & $\begin{array}{l}\text { capitulos } \\
\text { publicado } \\
\text { s }\end{array}$ & $\begin{array}{l}\text { Nuevos } \\
\text { capitulos }\end{array}$ & Divulgación & \\
\hline $\begin{array}{l}\text { Cap. } \\
\text { Libros }\end{array}$ & $70.14 \%$ & $70.50 \%$ & $70.00 \%$ & $14.12 \%$ & $71.25 \%$ & $69.53 \%$ & $60.9 \%$ \\
\hline
\end{tabular}

Fuente: Elaboración propia

La tabla 8 muestra la relación de las capacidades con congresos.

Tabla 8. Capacidades relacionadas con congresos realizados

\begin{tabular}{|c|c|c|c|c|c|c|c|}
\hline & $\begin{array}{l}\text { CV-Cap. } \\
\text { vigilar }\end{array}$ & $\begin{array}{l}\text { CR-Cap. } \\
\text { respuest } \\
\text { a }\end{array}$ & $\begin{array}{l}\text { CRP } \\
\text { Cap. } \\
\text { Resolver } \\
\text { problema } \\
\text { S }\end{array}$ & $\begin{array}{l}\text { CA - } \\
\text { Cap. } \\
\text { Aprende } \\
\text { r }\end{array}$ & $\begin{array}{l}\text { CI- } \\
\text { Cap. } \\
\text { innova } \\
\text { r }\end{array}$ & $\begin{array}{l}\text { CEC-Cap. } \\
\text { Explotación } \\
\text { del } \\
\text { conocimient } \\
\text { o }\end{array}$ & $\begin{array}{l}\text { TOTA } \\
\mathrm{L}\end{array}$ \\
\hline & $\begin{array}{l}\text { Asistenci } \\
\text { a de otros } \\
\text { centros }\end{array}$ & $\begin{array}{l}\text { Nuevos } \\
\text { congreso } \\
\text { S }\end{array}$ & congresos & $\begin{array}{l}\text { Ponencia } \\
\text { S }\end{array}$ & $\begin{array}{l}\text { Nuevos } \\
\text { temas }\end{array}$ & Divulgación & \\
\hline $\begin{array}{l}\text { Congreso } \\
\text { S }\end{array}$ & $72.5 \%$ & $71.50 \%$ & $70.62 \%$ & $74.12 \%$ & $61.25 \%$ & $69.53 \%$ & $70.42 \%$ \\
\hline
\end{tabular}

Fuente: Elaboración propia

El resultado de todas las relaciones se muestra en la tabla 9, donde se expresa el índice GVAPE final. 


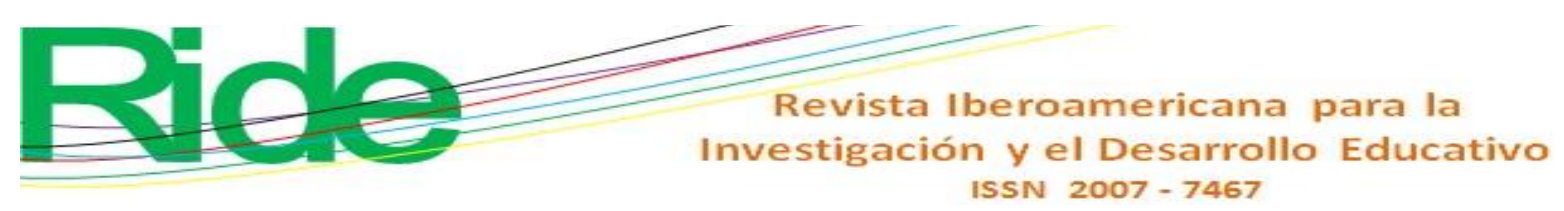

Tabla 9. El GVAPE final

\begin{tabular}{|l|l|}
\hline \multicolumn{2}{|l|}{ GVAPE } \\
\hline Proyectos & $80.6 \%$ \\
\hline Artículos & $68.32 \%$ \\
\hline Patentes & $24.31 \%$ \\
\hline Alumnos graduados & $72.81 \%$ \\
\hline Vinculaciones & $19.48 \%$ \\
\hline Capítulos de libro & $60.9 \%$ \\
\hline Congresos & $70.42 \%$ \\
\hline & \\
\hline GVAPE & $56.69 \%$ \\
\hline
\end{tabular}

Fuente: Elaboración propia

La tabla 10 muestra el cálculo de la generación de valor a partir de la cultura, aptitudes y comportamiento organizacional GVACUL.

Tabla 10. El GVACUL

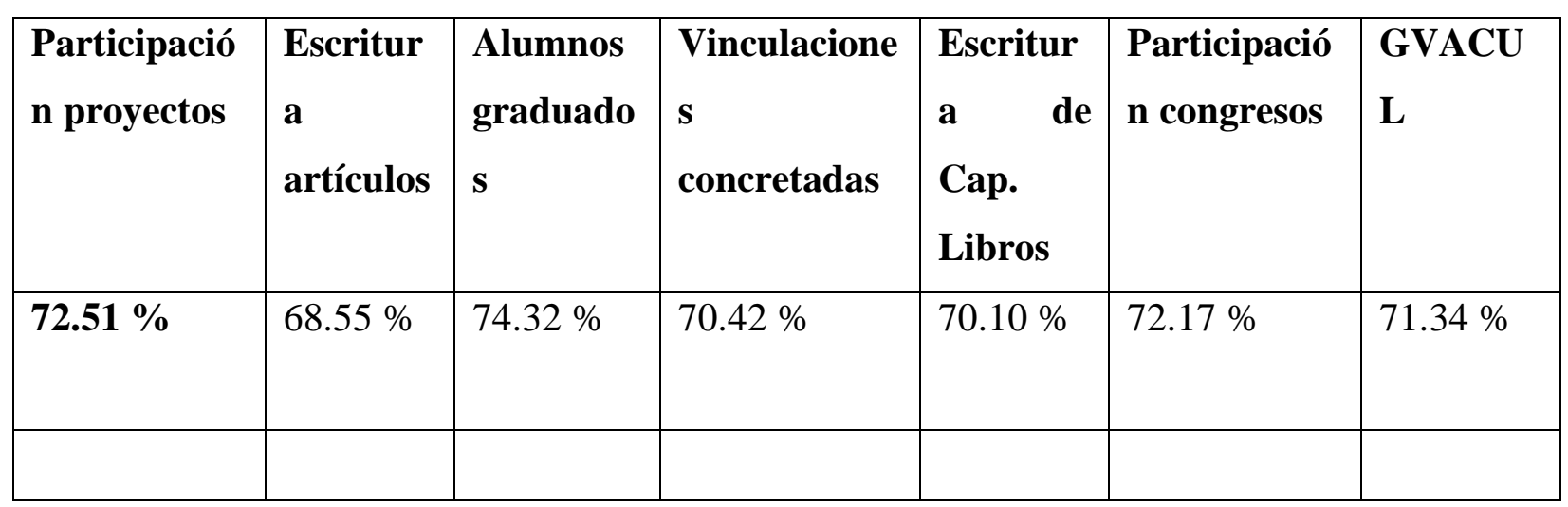

Fuente: Elaboración propia

La tabla 11 muestra el cálculo de la generación de valor a partir de las capacidades de la organización (GVACO). 


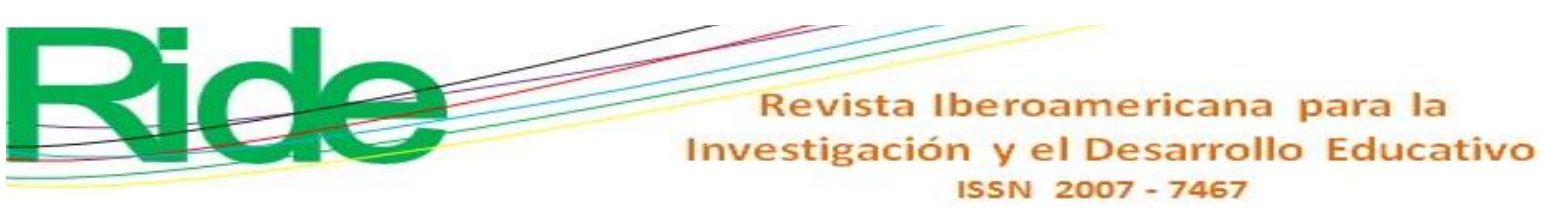

Tabla 11. El GVACO

\begin{tabular}{|l|l|l|l|l|l|l|}
\hline $\begin{array}{l}\text { CV-Cap. } \\
\text { vigilar }\end{array}$ & $\begin{array}{l}\text { CR-Cap. } \\
\text { respuesta }\end{array}$ & $\begin{array}{l}\text { CRP Cap. } \\
\text { Resolver } \\
\text { problemas }\end{array}$ & $\begin{array}{l}\text { CA -Cap. } \\
\text { Aprender }\end{array}$ & $\begin{array}{l}\text { CI-Cap. } \\
\text { Innovar }\end{array}$ & $\begin{array}{l}\text { CEC-Cap. } \\
\text { Explotación } \\
\text { del } \\
\text { conocimiento }\end{array}$ & GVACO \\
\hline $\mathbf{7 2 . 5 0} \%$ & $71.50 \%$ & $70.62 \%$ & $74.12 \%$ & $61.25 \%$ & $69.53 \%$ & $70.42 \%$ \\
\hline
\end{tabular}

Fuente: Elaboración propia

De esta forma, el IMIO se determinará en función de estos cuatro valores, de acuerdo con la ecuación 1.

$$
I M I O=f \frac{(70.42 \%+56.69 \%+71.12 \%+71.34 \%)}{4}=\frac{269.57}{4}=67.39 \%
$$

\section{Discusión}

Los resultados de este estudio destacan que las instituciones deben trabajar y dar ayuda a sus investigadores para que elaboren vinculaciones y patentes, así como para reforzar la publicación de artículos y capítulos de libro. Se muestra que en el campo de la difusión, los valores obtenidos son interesantes, ya que la divulgación sí se realiza de manera sistemática.

Finalmente, un aspecto relevante en los resultados del índice de memoria de inteligencia organizacional (IMIO) para el total de la muestra de las instituciones participantes del estudio es que el elemento "capacidades" muestra el menor grado de generación de valor para la dinámica de ellas. Ello significa que aunque hay percepción positiva de la importancia del conocimiento en el contexto de las instituciones, en definitiva resulta más de criterio que de acciones concretas orientadas a desarrollar competencias. De acuerdo con la teoría sobre gestión del conocimiento, no basta con identificar, adquirir o crear conocimiento, sino que es más importante tener las capacidades o competencias para la respectiva apropiación, creación y uso del conocimiento, para la propia generación de valor agregado, y así generar ventajas competitivas.

Es importante destacar la necesidad de una perspectiva cualitativa y cuantitativa en un mayor nivel de integración sistémica para la presente aportación. Asimismo, corresponde a futuros trabajos el diseño, la aplicación y la retroalimentación (mejora), lo que llevará, sin duda, al aprendizaje en todas las dimensiones y al fortalecimiento de la perspectiva de la gestión de conocimiento. 


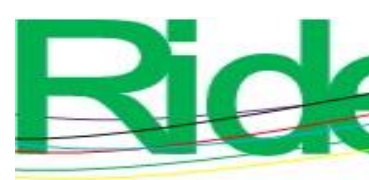

Revista Iberoamericana para la Investigación y el Desarrollo Educativo

ISSN $2007-7467$

Por otra parte, con la finalidad de evaluar los resultados en relación con otros documentos similares, dos estudios fueron localizados. El primero es el de Lobato-Baez, Morales-Rosales, Toriz-Palacios y López-Arciga (2018), quienes proponen la construcción de un modelo de gestión del conocimiento para la industria del software en México. En este trabajo recopilan indicadores a partir de la aplicación de la matriz de índice de medición de inteligencia organizacional (IMIO). Los autores evaluaron la viabilidad y midieron las mejoras que se alcanzaron en un periodo de cuatro meses en una pyme de Tlaxcala (México), lo que permitirá capturar, almacenar, recuperar, gestionar y administrar el conocimiento para contribuir a la disminución de costos para mejorar la comunicación de la calidad de servicio, entre otros.

La segunda investigación es la de Bernal, Torres, Turriago y Sierra (2010), quienes miden la gestión del conocimiento a 1168 personas de 48 organizaciones participantes con la matriz IMIO. En este trabajo se obtuvo un grado de gestión del conocimiento (GGC) equivalente a 72.10\%, es decir, una gestión buena del conocimiento. Para el caso de la presente investigación, se tiene una desventaja de un poco más de $4 \%$.

La limitación más importante a lo largo del estudio es la falta de disponibilidad de las instituciones de la muestra. Los trabajos futuros se centran en ampliar el tamaño de la muestra y en el diseño y validación (a través de simuladores) de mejoras.

\section{Conclusiones}

En general, desde el inicio del presente siglo, en México el desarrollo acelerado de las tecnologías de la información y las comunicaciones y la complejidad adquirida por la internet han provocado que la sociedad esté más interconectada y que las organizaciones educativas se tornen cada vez más complejas. Esto ha provocado una reiterada inquietud por parte de la comunidad académica para propiciar actividades relacionadas con la capacitación y la investigación en el campo de la gestión del conocimiento y sus relaciones.

Los directivos de instituciones educativas de posgrado o de investigación han comenzado a incluir en sus planes de desarrollo estrategias relacionadas con la gestión del conocimiento, como factor generador de valor para su capacidad competitiva. El gobierno de México, desde hace dos sexenios, ha estado formulando agendas nacionales, regionales y locales, en donde la gestión del conocimiento es considerada prioritaria para el desarrollo y la competitividad de cada comunidad.

Para el caso que se analiza en esta investigación, se observa que en general — según los resultados expresados por los encuestados- existe interés y un significativo grado de gestión del 


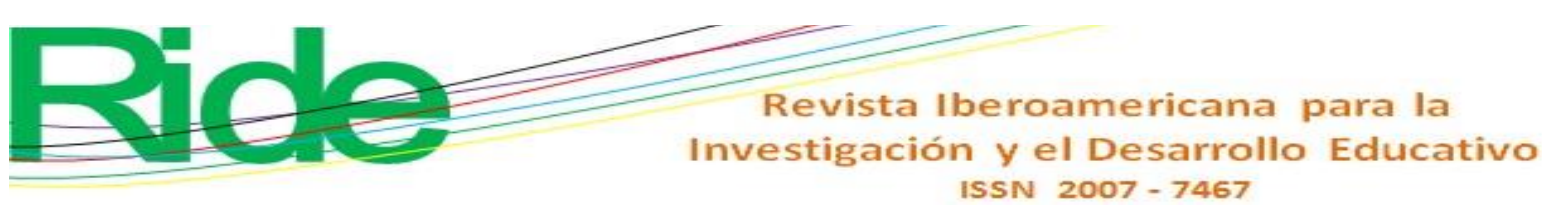

conocimiento por parte de las instituciones encuestadas, donde la capacitación de las personas, el análisis del entorno, el uso de la tecnologías de la información y las comunicaciones, la cultura, las actitudes y el comportamiento, así como la memoria organizacional juegan un rol importante para la dinámica y para brindar mejores resultados en esta tarea tan importante, que es la investigación.

\section{Referencias}

Aguilera, A. y Riascos, S. C. (2009). Direccionamiento estratégico apoyado en las TIC. Estudios Gerenciales, 25(111), 127-143.

Alfonzo, M (2018). Gestión del conocimiento e instituciones educativas.Educ@ción en Contexto, 4(8), 158-177.

Alzate, O. F. (2015). La gestión del conocimiento un desafío para las instituciones educativas en Colombia: emergencias y tensiones desde la teoría del capital intelectual. Rev. Gestión de la Educación, 5(2), 137-150.

Albornies Ortiz Ángel y Áldazabal Basauri Jon. Mik s. Coop. Sussex university. España. 2005

Avendaño-Villa, I., Cortés-Peña, O. F. y Guerrero-Cuentas, H. (2015). Competencias sociales y tecnologías de la información y la comunicación como factores asociados al desempeño en estudiantes de básica primaria con experiencia de desplazamiento forzado. Revista Diversitas: Perspectivas en Psicología, 11(1), 13-36.

Barroso, F. (2011). Gestión del conocimiento en instituciones de educación superior y centros de investigación científica en el estado de Yucatán. XVI Congreso Internacional de Contaduría, Administración e Informática. Octubre 5, 6 y 7 de 2011, Ciudad Universitaria, CDMX.

Begoña, G. S. (2008). Tendencias actuales de la investigación en docencia universitaria. Edusfarm, Revista d'Educació Superior en Farmàcia, (1), 1-13.

Bernal, T. C., Torres, Turriago, Á. H. y Sierra, A. H. (2010). Aproximación a la medición de la gestión del conocimiento empresarial. AD-MINISTE, (16), 30-49.

Castells, M. (1997). The Rise of the Network Society. Chichester, West Sussex. United Kingdom: Wiley-Blackwell.

Coll, C. (2009). Aprender y enseñar con las TIC: expectativas, realidad y potencialidades. En Carneiro, R., Toscano, J. C. y Díaz, T. (comps.), Los desafíos de las TIC para el cambio educativo (pp. 113-126). Madrid: OEI-Santillana, Fundación Santillana. 
Racionero, S. y Serradell, O. (2005). Antecedentes de las comunidades de aprendizaje. Educar, 35, 29-39.

Representación de la Unesco en Perú (2011). Manual de gestión para directores instituciones educativas. Lima, Perú: Ministerio de Educación.

Rubinstein, G. (2005). Knowledge Creation. In Encyclopedia of Social Measurement. Elsevier Science Publishing Co Inc.

Spooren, P., Mortelmans, D. and Denekens, J. (2007). Student evaluation of teaching quality in higher education: development of an instrument based on 10 Likert scales. Assessment \& Evaluation in Higher Education, 32(6), 667-679.

Valdivieso, S. (2001). North y el cambio histórico: luces y sombras de la nueva historia institucional. Revista de Economía Institucional, 3(4), 157-172. 


\begin{tabular}{|l|l|}
\hline Rol de Contribución & Autor (es) \\
\hline Conceptualización & Jesús Antonio Álvarez Cedillo. \\
\hline Metodología & Brenda García Jarquín. \\
\hline Software & Teodoro Álvarez-Sánchez. \\
\hline Validación & Mario Aguilar Fernández. \\
\hline Análisis Formal & $\begin{array}{l}\text { Jesús Antonio Álvarez Cedillo ,Teodoro Álvarez Sánchez } \\
\text { (IGUAL). }\end{array}$ \\
\hline Investigación & $\begin{array}{l}\text { Jesús Antonio Álvarez Cedillo ,Teodoro Álvarez Sánchez } \\
\text { (IGUAL). }\end{array}$ \\
\hline Recursos & Julián Patiño Ortiz. \\
\hline Curación de datos & Mario Aguilar Fernández. \\
\hline $\begin{array}{l}\text { Escritura - Preparación del } \\
\text { borrador original }\end{array}$ & Jesús Antonio Álvarez Cedillo \\
\hline $\begin{array}{l}\text { Escritura } \quad \text { Revisión } \\
\text { edición }\end{array}$ & Julián Patiño Ortiz \\
\hline Visualización & Mario Aguilar Fernández \\
\hline Supervisión & Jesús Antonio Álvarez Cedillo \\
\hline $\begin{array}{l}\text { Administración } \\
\text { Proyectos }\end{array}$ & Jesús Antonio Álvarez Cedillo \\
\hline Adquisición de fondos & Brenda García Jarquín \\
\hline
\end{tabular}

